\title{
Architectural Changes in the Medial Gastrocnemius on Sonography after Nerve Ablation in Healthy Adults
}

\author{
Jae Eun Park ${ }^{1}$, Yeon-Jae Seong², Eun Sang Kim², Dongho Park ${ }^{1}$, \\ Yonghyun Lee ${ }^{1}$, Hyerin Park ${ }^{1}$, and Dong-wook Rha ${ }^{1}$ \\ ${ }^{1}$ Department and Research Institute of Rehabilitation Medicine, Yonsei University College of Medicine, Seoul; \\ ${ }^{2}$ Hafis Clinic, Seoul, Korea.
}

\begin{abstract}
Architectural changes in healthy muscle after denervation have not yet been reported. This study aimed to investigate architectural changes in the medial head of the gastrocnemius muscle (GCM) after aesthetic tibial nerve ablation in healthy adults using ultrasonography (US). The effects of tibial nerve ablation were verified by visual observation and surface electromyography analysis. US images of medial GCMs were taken by one trained physician using B-mode and real-time US with a linear-array probe before nerve ablation, at 1 week after nerve ablation and at 3 months after nerve ablation in an anatomic standing position with the feet about shoulder-width apart in 19 healthy adults ( 17 females and 2 males). Muscle thickness was significantly reduced on the left side at 1 week and 3 months after the procedure and on the right side at 3 months after the procedure $(p<0.050)$. Although fascicle length was not significantly changed, pennation angle was significantly reduced on both sides at 3 months after the procedure $(p<0.050)$. Muscle thickness and pennation angle of the muscle fascicle were significantly reduced, although fascicle length was not significantly changed, after tibial nerve ablation in the medial GCM of healthy adults.
\end{abstract}

Key Words: Pennation angle, fascicle length, muscle thickness, nerve ablation, ultrasonography, muscle architecture

Various diseases, including stroke, spinal cord injury, and cerebral palsy (CP), may result in central or peripheral nerve injuries and can cause denervation of muscles. Denervation of muscles results in muscle atrophy, decreased muscle strength, and diminished locomotor abilities. Denervation of muscles can also cause changes in muscle architecture. ${ }^{1}$ Muscle architecture is an important determinant of muscle function ${ }^{2}$ and is defined as the arrangement of muscle fibers within a muscle relative to the axis of force generation. ${ }^{3}$ Muscle architecture refers to bundles of fibers known as fascicles and is described by fascicle lengths (FLs) and pennation angles (PAs). ${ }^{4}$

Received: May 7, 2019 Revised: July 9, 2019

Accepted: July 17, 2019

Corresponding author: Dong-wook Rha, MD, PhD, Department and Research Institute of Rehabilitation Medicine, Yonsei University College of Medicine, 50-1 Yonsei-ro, Seodaemun-gu, Seoul 03722, Korea.

Tel: 82-2-2228-3717, Fax: 82-2-363-2795, E-mail: medicus@yuhs.ac

-The authors have no potential conflicts of interest to disclose.

(c) Copyright: Yonsei University College of Medicine 2019

This is an Open Access article distributed under the terms of the Creative Commons Attribution Non-Commercial License (https://creativecommons.org/licenses/ by-nc/4.0) which permits unrestricted non-commercial use, distribution, and reproduction in any medium, provided the original work is properly cited.
Also contributing to muscle architecture are muscle-tendon properties, which are associated with functional performance. ${ }^{5,6}$

Ultrasonography (US) has become a popular method for characterizing muscle architecture because of its safety and noninvasive nature. ${ }^{7}$ The reliability of muscle architecture measurements by B-mode ultrasound imaging has been demonstrated in previous studies. ${ }^{8,9}$ Using US, changes in the muscle architecture of children with $\mathrm{CP}$ were reported after botulinum neurotoxin (BoNT) injection, ${ }^{10}$ and muscle architecture was compared between the paretic limbs and non-paretic limbs in chronic stroke patients. ${ }^{11}$

To the best of our knowledge, architectural changes of healthy muscle without any comorbid disease after denervation have not yet been reported. This study aimed to investigate architectural changes in the gastrocnemius muscles (GCMs) after aesthetic tibial nerve ablation in healthy adults using US. This was a prospective study conducted in a university-affiliated hospital. Ethical approval was granted by Yonsei University College of Medicine institutional review board and ethics committee (4-2016-0505).

We recruited 19 healthy adults who were scheduled to undergo aesthetic volume reduction of the medial GCM. Sub- 
jects were excluded if they had a previous history of (a) central nervous system disease, (b) neuromuscular disease, or (c) any surgical procedures at examined extremities, such as BoNT injection and liposuction. In total, 19 adults ( 17 females and 2 males) with ages ranging from 19-48 years $(28.68 \pm 7.99)$ were enrolled. Thirty-eight limbs from 19 healthy adults underwent aesthetic tibial nerve ablation.

US was used to identify the location of nerves and vessels in the popliteal fossa and calf area, with the subject lying in a prone position. After local anesthesia using $1 \%$ lidocaine, a radio frequency probe connected to a $400-\mathrm{kHz}$ electrical surgical unit (ITC-300D; ITC, Daejeon, Korea) was inserted through a small incision in the skin below the popliteal crease. After finding the exact location of the motor nerve branches to the medial GCM with electrical nerve stimulation and placing the tip of the probe on the nerve, radio frequency energy was transmitted to ablate the motor nerve branches innervated to the medial GCM. The surface of the radio frequency probe was insulated, except for the tip area, so that we could ablate nerve branches precisely with minimal injury to adjacent tissues. After the procedure, we confirmed that the nerve ablation was successful when the maximum root mean square (RMS) value of electromyography of the GCM decreased to at least $30 \%$ of the maximum pre-ablation value during walking. ${ }^{12}$ We recorded surface electromyography (MA300-XVI, Motion lab systems, USA; sampling frequency $=1000 \mathrm{~Hz}$ ) with the electrodes placed on the medial head of the GCM. RMS values were calculated for individual muscles. Pre- and post-procedure RMS values are expressed as a ratio of the maximum pre-

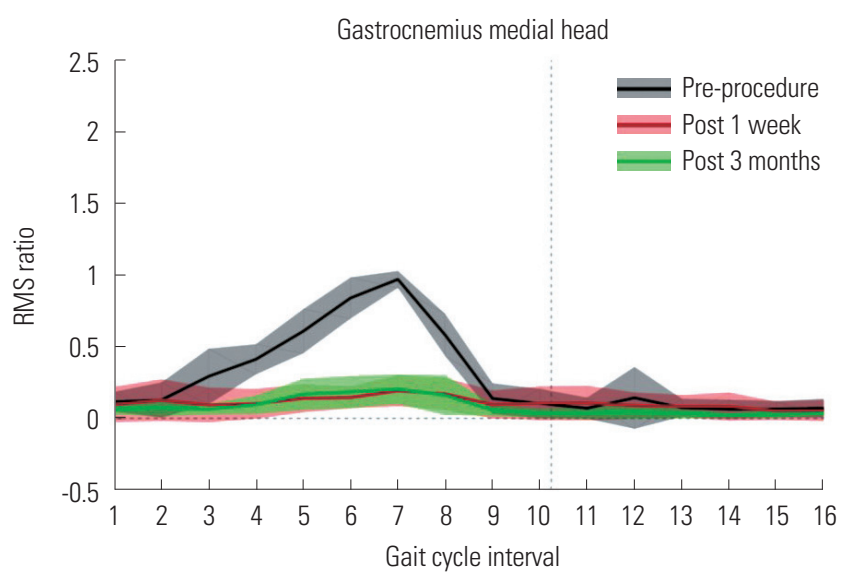

Fig. 1. Root mean square (RMS) ratio values of the medial head of the gastrocnemius muscles. $\mathrm{X}$-axis indicates that one gait cycle divided into 16 equally spaced intervals.

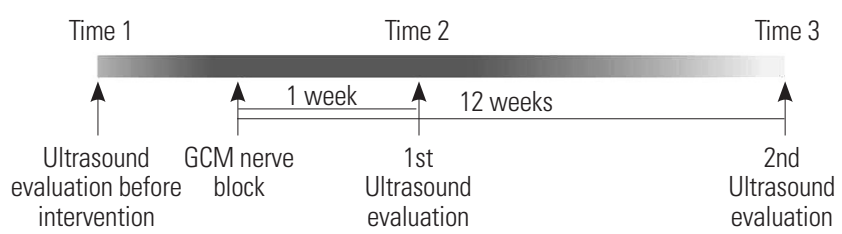

Fig. 2. Time table. GCM, gastrocnemius muscle. procedure RMS value (Fig. 1).

US images of medial GCMs were taken by a single trained physician using B-mode and real-time US (Accuvix V10c system; Samsung Medison, Seoul, Korea) with a linear-array probe (5-12 MHz) before nerve ablation, at 1 week after nerve ablation, and at 3 months after nerve ablation in an anatomical standing position with the feet approximately shoulderwidth apart (Figs. 2 and 3). US images were taken within 15 minutes per patient.

Muscle thickness (MT) was measured as the longest distance between the superficial and the deep fascia of the GCM in a longitudinal US image. Muscle FL was defined as the straight-line distance between the superficial and deep muscular fascia parallel to the lines of the collagenous tissue visible on the image. The PA was defined as the angle made between the upper fascia and the direction of the muscle fascicles (Fig. 4).

Statistical analyses were performed using SAS statistical software, version 9.4 (SAS Institute, Cary, NC, USA). A linear mixed model was applied to analyze data from repeated measurements. Parameters after tibial nerve ablation were compared

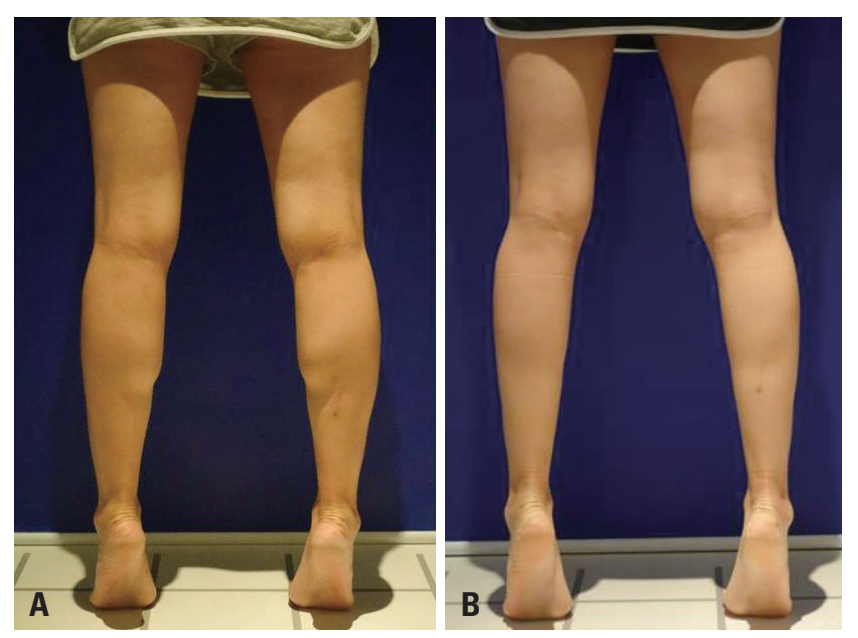

Fig. 3. Gross photos of gastrocnemius muscles (GCMs). Subjects were asked to elevate the heel to contract the GCM. (A) Gross photo taken before tibial nerve block. (B) Gross photo taken three weeks after the tibial nerve block. The size of the GCMs has decreased.

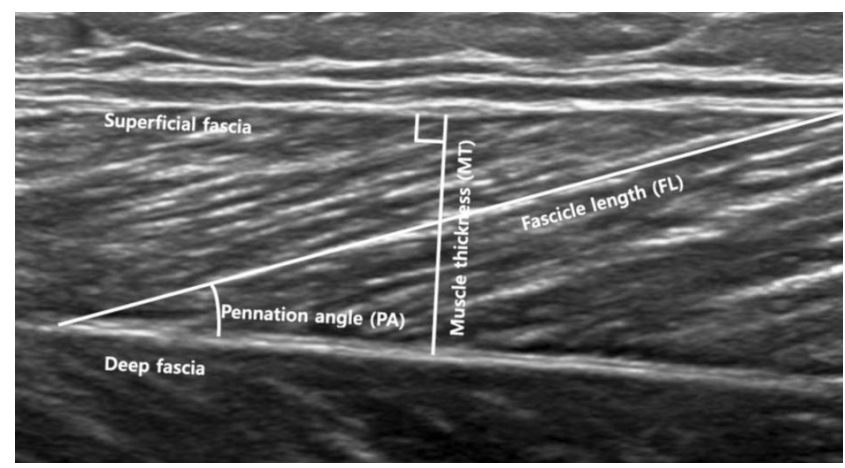

Fig. 4. Ultrasound image showing muscle architecture parameters measured. 
to those before intervention to determine whether there were changes in muscle architecture. $p<0.050$ was considered statistically significant.

The clinical features of the participants are shown in Table 1. MT of the medial GCM was significantly reduced on the left side at 1 week and 3 months after tibial nerve ablation and on the right side 3 months after tibial nerve ablation $(p<0.050)$. Although the FLs of the medial GCM were not significantly changed, the PAs of the medial GCM were significantly reduced on both sides at 3 months after tibial nerve ablation $(p<0.050)$ (Table 2, Fig. 5).

Significant reductions in muscle volumes of paretic limbs in individuals with central nervous system lesions from CP and peripheral nervous system lesions from post-traumatic peripheral neuropathy have been demonstrated by magnetic resonance imaging in previous studies. ${ }^{13,14}$ An in vivo US image study has also been successfully conducted in studies of muscular geometric architecture in humans. A US image study is faster, more convenient, and less expensive for assessing muscular architecture. According to previous reports, the architectural characteristics of muscle structures can be determined using

Table 1. Characteristics of Participants

\begin{tabular}{lc}
\hline & Values \\
\hline Age $(\mathrm{yr})$ & $28.68 \pm 7.99(19-48)$ \\
Sex $(\mathrm{n}, \%)$ & $2(10.53)$ \\
$\quad$ Male & $17(89.47)$ \\
\hline Female & $162.04 \pm 4.84(155-178)$ \\
Height (cm) & $57.27 \pm 7.26(47-69)$ \\
Body weight (kg) & \\
Left & $2.23 \pm 0.24(1.73-2.53)$ \\
\hline Thickness & $9.77 \pm 13.70(4.83-66.20)$ \\
\hline Fascicle & $20.11 \pm 2.82(15.30-27.40)$ \\
\hline Pennation & \\
Right & $2.24 \pm 0.22(1.71-2.65)$ \\
\hline Thickness & $6.86 \pm 0.87(5.19-8.36)$ \\
\hline Fascicle & $19.37 \pm 2.43(16.50-25.20)$ \\
\hline Pennation & \\
\hline
\end{tabular}

Values are expressed as a mean \pm standard deviation (range) or number (\%). conventional grayscale brightness mode (B-mode) ultrasound imaging to assess components of muscle morphology (structure) and morphometry (size) in exploration of muscle function. ${ }^{15-18}$ Musculoskeletal US is considered a potential tool for quantifying muscle architectural changes in vivo, revealing the arrangement of muscle fibers within a muscle. ${ }^{4}$

Architectural parameters, such as FL and PA, affect muscle functions, as well as intrinsic factors, such as fiber composition. ${ }^{19,20}$ In previous studies, human muscle architecture was observed in cadaver specimens and suggested to be related to functional characteristics, ${ }^{21-24}$ although very little data has been reported for healthy human muscles. FL is indicative of the number of sarcomeres in a series, whereas PA reflects the positive angle between muscle fibers and muscle aponeurosis. ${ }^{4}$ Muscle fiber length is the primary determinant of muscle excursion and is proportional to the maximum excursion of the muscle and velocity of contraction. Therefore, the fiber length to muscle length ratio is a good indicator of the design of skeletal muscle, either for producing high force or high excursion..$^{25,26}$ Unfortunately, the only method to determine muscle fiber length in muscle is to obtain whole, fixed muscles and to dissect individual fibers or fiber bundles from these fixed muscles. Most studies have measured the length of a small bundle of fibers, and thus, most of these lengths were actually FLs. The PA gives information about muscle strength, and the larger the PA, the more contractile material can be packed within a certain volume to increase the physiological cross sectional area of the muscle. ${ }^{27}$ An increase in PA therefore indicates an increase in the muscle's capacity to produce force. ${ }^{4,27}$

The present study showed that MT and PA for both sides were significantly decreased, whereas the FL for both sides was not significantly changed, in the medial GCMs of subjects in an anatomical standing position after tibial nerve ablation. Some previous studies have reported that toxin injection into healthy muscles induces muscle atrophy in animals ${ }^{28-31}$ and humans, ${ }^{13}$ although muscle architectural changes were not reported. In pathological studies, architectural changes after denervation have been analyzed using musculoskeletal US. The brachialis muscle showed shorter FL and the PA was larger in the affected side, compared to the unaffected side, in patients

Table 2. Changes in Muscle Architecture

\begin{tabular}{lccc}
\hline & Time $\mathbf{1}$ (baseline) & Time 2 (1 week) & Time 3 (12 weeks) \\
\hline Left & & & $1.69 \pm 0.27(1.29-2.23)^{*}$ \\
\hline Thickness (cm) & $2.23 \pm 0.24(1.73-2.53)$ & $2.12 \pm 0.27(1.64-2.58)^{*}$ & $6.60 \pm 1.04(5.13-8.34)$ \\
\hline Fascicle length (cm) & $6.64 \pm 1.06(4.83-9.34)$ & $6.92 \pm 0.99(5.50-8.85)$ & $15.10 \pm 2.80(10.60-21.00)^{*}$ \\
\hline Pennation angle (degrees) & $20.10 \pm 2.80(15.30-27.40)$ & $18.60 \pm 3.30(13.60-25.50)$ & $1.70 \pm 0.29(1.07-2.29)^{*}$ \\
Right & & & $6.55 \pm 0.85(5.28-8.06)$ \\
\hline Thickness (cm) & $2.24 \pm 0.22(1.71-2.65)$ & $2.17 \pm 0.30(1.53-2.60)$ & $15.00 \pm 2.50(10.20-18.10)^{*}$ \\
\hline Fascicle length (cm) & $6.86 \pm 0.87(5.19-8.36)$ & $6.89 \pm 0.82(5.91-8.71)$ & $18.80 \pm 3.10(12.90-24.50)$ \\
\hline Pennation angle (degrees) & $19.40 \pm 2.40(16.50-25.20)$ & & \\
\hline
\end{tabular}

Values are expressed as a mean \pm standard deviation (range).

${ }^{*} p<0.050$ vs. Time 1 in linear mixed model. 

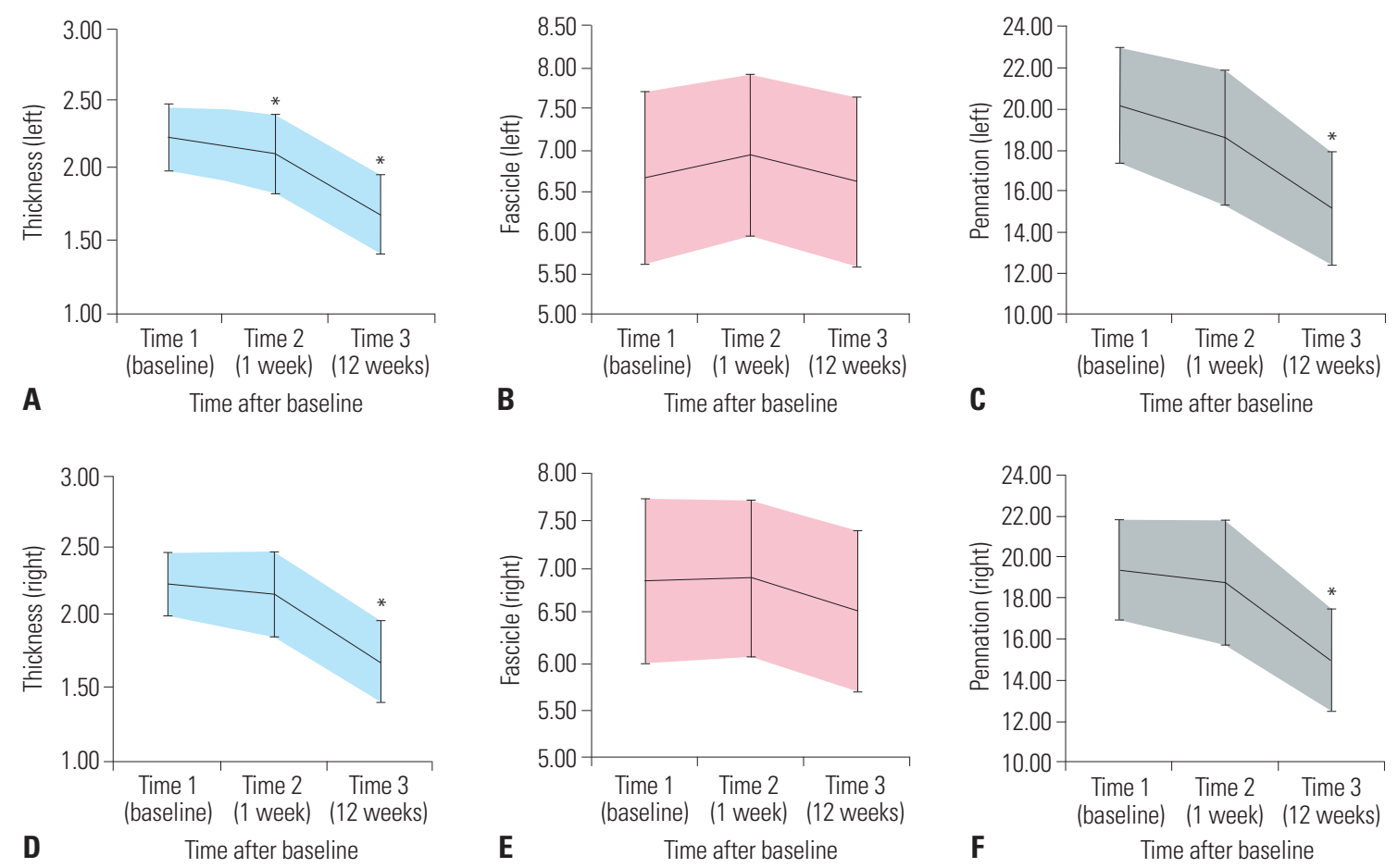

Fig. 5. Changes in muscle architecture parameters after tibial nerve block. Changes of (A) left gastrocnemius muscle (GCM) thickness, (B) left GCM fascicle length, (C) left GCM pennation angle, (D) right GCM thickness, (E) right GCM fascicle length and (F) right GCM pennation angle. Bars indicate one standard deviation. ${ }^{*} p<0.050$ vs. baseline (post-hoc test).

with stroke. ${ }^{11}$ Decreases in MT and PA and increases in FL were shown in the hemiplegic side of stroke patients at 2 months after BoNT-A injection when US examination was performed in the resting ankle position with knee extension. ${ }^{32}$ In children with spastic $\mathrm{CP}$, the MT and PA were significantly decreased, whereas the FL was increased, at 1 and 3 months after BoNT-A injection in the resting ankle position. However, there was no significant change in FL in the neutral ankle position..$^{10}$ Another recent study reported that PA decreased, while FL increased, at 12 weeks after BoNT-A injection in children with CP both in the resting and the maximum dorsiflexion position of the ankle. ${ }^{33}$ However, the changes in FL at the neutral ankle position was not reported in that study.

The above-mentioned changes in denervated FL could not be compared to our study because we performed US only in the neutral ankle angle (anatomical standing position), not in the resting ankle and the maximum dorsiflexion position of the ankle. As participants still had pain at 1 week after the procedure, maximum ankle DF and relaxed resting ankle position were difficult to maintain. Therefore, we used only a neutral ankle position.

While our study revealed no significant difference in FL, there were differences in PA before and after nerve ablation in the neutral ankle angle. This is similar to the results of a previous study in children with CP after BoNT-A injection. ${ }^{10}$ This suggests that the architectural changes of muscles after nerve ablation in healthy muscles were similar to those after BoNT-A injection in spastic muscles at a neutral ankle angle. Although a neutral ankle angle has an advantage of being able to measure and compare the muscle architecture more reliably, possible differences in architectural and physiological characteristics at a resting ankle angle between healthy and spastic muscles should also be considered. There has been few studies, however, to determine whether the resting sarcomere length of a spastic muscle is shortened, lengthened, or unchanged. If the resting sarcomere length is shortened in a spastic muscle due to pathological hypertonus, it might be lengthened after BoNT-A injection and the FL might be lengthened in the resting position. However, we could not study FL in a resting ankle position after nerve ablation in healthy muscle. In addition, we do not know how sarcomere length may change after denervation. Further study is needed to evaluate the microstructural changes (e.g., sarcomere length) after denervation in both healthy and spastic muscles that cannot be measured using US.

Previous studies demonstrated a significant relationship between $\mathrm{MT}$ and $\mathrm{PA},{ }^{27}$ as well as a significant decrease in the PA of healthy young adults in response to disuse. ${ }^{34,35}$ Both muscle atrophy and a decrease in PA of the vastus lateralis muscles may be contributing factors to the decreased force production of the quadriceps observed in $\mathrm{CP}^{4}$ The decreases in the PA and MT after nerve ablation in our study suggest a negative influence of denervation on the ability of healthy muscle to produce force.

This study was the first study to report on architectural changes of the GCM after tibial nerve ablation in healthy adults. The MT and PA of the muscle fascicle of the medial head of GCM 
were significantly reduced, although FL was not significantly changed in the anatomical standing position. Muscle architecture is a key determinant of muscle power and function. Further studies are therefore needed to determine the effect of structural changes of healthy muscles after nerve ablation on muscle power and function.

There were some limitations in our study. First, we only measured FL using US. As we did not evaluate the microscopic structure of the muscles, we could not determine changes in the number or length of sarcomeres: it is still unclear whether there are changes in the number or length of sarcomere after nerve ablation. Second, most of the subjects were females, and sex differences might have been a limitation. A previous study reported sex differences in muscle architecture in human soleus and GCMs in healthy adults. ${ }^{36}$ Third, we only assess changes in the neutral ankle position, and muscle architecture at various ankle angles was not analyzed. These limitations should be addressed in future studies.

\section{ACKNOWLEDGEMENTS}

This study is supported by a research grant from the Research Institute of Rehabilitation Medicine, Yonsei University College of Medicine for 2017.

\section{AUTHOR CONTRIBUTIONS}

Conceptualization: Dong-wook Rha. Data curation: Jae Eun Park. Formal analysis: Jae Eun Park, Dongho Park, Yonghyun Lee, and Hyerin Park. Investigation: Jae Eun Park. Methodology: Dong-wook Rha. Project administration: Dong-wook Rha. Resources: All authors. Software: Jae Eun Park, Dongho Park, and Dong-wook Rha. Supervision: Dong-wook Rha. Validation: Yeon-Jae Seong and Eun Sang Kim. Visualization: Jae Eun Park and Dong-wook Rha. Writing-original draft: Jae Eun Park. Writing_review \& editing: Jae Eun Park and Dong-wook Rha.

\section{ORCID iDs}

Jae Eun Park Yeon-Jae Seong Eun Sang Kim Dongho Park Yonghyun Lee Hyerin Park Dong-wook Rha https://orcid.org/0000-0001-8790-7681 https://orcid.org/0000-0002-8061-6352 https://orcid.org/0000-0002-7871-974X https://orcid.org/0000-0002-9308-2222 https://orcid.org/0000-0002-0997-3672 https://orcid.org/0000-0001-5359-4381 https://orcid.org/0000-0002-7153-4937

\section{REFERENCES}

1. Sunderland S, Ray LJ. Denervation changes in mammalian striated muscle. J Neurol Neurosurg Psychiatry 1950;13:159-77.

2. Gans C. Fiber architecture and muscle function. Exerc Sport Sci Rev 1982;10:160-207.

3. Lieber RL. Skeletal muscle structure and function: implications for rehabilitation and sports medicine. 1st ed. Baltimore: Williams \& Wilkins; 1992.

4. Moreau NG, Teefey SA, Damiano DL. In vivo muscle architecture and size of the rectus femoris and vastus lateralis in children and adolescents with cerebral palsy. Dev Med Child Neurol 2009;51: 800-6.

5. Lebiedowska MK, Gaebler-Spira D, Burns RS, Fisk JR. Biomechanic characteristics of patients with spastic and dystonic hypertonia in cerebral palsy. Arch Phys Med Rehabil 2004;85:875-80.

6. Aiona M. Muscle length and force production in patients with cerebral palsy-the effects of tendon lengthening. In: IEEE, editor. Pediatric gait: a new millennium in clinical care and motion analysis technology. 1st ed. Chicago: IEEE; 2000. p.30-8.

7. Narici M. Human skeletal muscle architecture studied in vivo by non-invasive imaging techniques: functional significance and applications. J Electromyogr Kinesiol 1999;9:97-103.

8. Narici MV, Binzoni T, Hiltbrand E, Fasel J, Terrier F, Cerretelli P. In vivo human gastrocnemius architecture with changing joint angle at rest and during graded isometric contraction. J Physiol 1996; 496(Pt 1):287-97.

9. Blazevich AJ, Cannavan D, Coleman DR, Horne S. Influence of concentric and eccentric resistance training on architectural adaptation in human quadriceps muscles. J Appl Physiol (1985) 2007; 103:1565-75.

10. Park ES, Sim E, Rha DW, Jung S. Architectural changes of the gastrocnemius muscle after botulinum toxin type A injection in children with cerebral palsy. Yonsei Med J 2014;55:1406-12.

11. Li L, Tong KY, Hu X. The effect of poststroke impairments on brachialis muscle architecture as measured by ultrasound. Arch Phys Med Rehabil 2007;88:243-50.

12. Romkes J, Hell AK, Brunner R. Changes in muscle activity in children with hemiplegic cerebral palsy while walking with and without ankle-foot orthoses. Gait Posture 2006;24:467-74.

13. Schroeder AS, Ertl-Wagner B, Britsch S, Schröder JM, Nikolin S, Weis J, et al. Muscle biopsy substantiates long-term MRI alterations one year after a single dose of botulinum toxin injected into the lateral gastrocnemius muscle of healthy volunteers. Mov Disord 2009;24:1494-503.

14. Fleckenstein JL, Watumull D, Conner KE, Ezaki M, Greenlee RG Jr, Bryan WW, et al. Denervated human skeletal muscle: MR imaging evaluation. Radiology 1993;187:213-8.

15. Hides JA, Cooper DH, Stokes MJ. Diagnostic ultrasound imaging for measurement of the lumbar multifidus muscle in normal young adults. Physiother Theory Pract 1992;8:19-26.

16. Hides J, Richardson C, Jull G, Davies S. Ultrasound imaging in rehabilitation. Aust J Physiother 1995;41:187-93.

17. Stokes M, Hides J, Nassiri DK. Musculoskeletal ultrasound imaging: diagnostic and treatment aid in rehabilitation. Physical Therapy Reviews 1997;2:73-92.

18. Whittaker JL, Teyhen DS, Elliott JM, Cook K, Langevin HM, Dahl $\mathrm{HH}$, et al. Rehabilitative ultrasound imaging: understanding the technology and its applications. J Orthop Sports Phys Ther 2007; 37:434-49.

19. Burkholder TJ, Fingado B, Baron S, Lieber RL. Relationship between muscle fiber types and sizes and muscle architectural properties in the mouse hindlimb. J Morphol 1994;221:177-90.

20. Sacks RD, Roy RR. Architecture of the hind limb muscles of cats: functional significance. J Morphol 1982;173:185-95.

21. An KN, Hui FC, Morrey BF, Linscheid RL, Chao EY. Muscles across the elbow joint: a biomechanical analysis. J Biomech 1981;14:65969 .

22. Brand RA, Pedersen DR, Friederich JA. The sensitivity of muscle force predictions to changes in physiologic cross-sectional area. J Biomech 1986;19:589-96.

23. Edgerton VR, Apor P, Roy RR. Specific tension of human elbow flexor muscles. Acta Physiol Hung 1990;75:205-16. 
24. Huijing PA. Architecture of the human gastrocnemius muscle and some functional consequences. Acta Anat (Basel) 1985;123:101-7.

25. Lieber RL, Steinman S, Barash IA, Chambers H. Structural and functional changes in spastic skeletal muscle. Muscle Nerve 2004; 29:615-27.

26. Lieber RL, Fridén J. Functional and clinical significance of skeletal muscle architecture. Muscle Nerve 2000;23:1647-66.

27. Kawakami Y, Abe T, Fukunaga T. Muscle-fiber pennation angles are greater in hypertrophied than in normal muscles. J Appl Physiol (1985) 1993;74:2740-4.

28. Manske SL, Boyd SK, Zernicke RF. Muscle and bone follow similar temporal patterns of recovery from muscle-induced disuse due to botulinum toxin injection. Bone 2010;46:24-31.

29. Shen J, Ma J, Lee C, Smith BP, Smith TL, Tan KH, et al. How muscles recover from paresis and atrophy after intramuscular injection of botulinum toxin A: study in juvenile rats. J Orthop Res 2006;24: 1128-35.

30. Glass GE, Hussain M, Fleming AN, Powell BW. Atrophy of the intrinsic musculature of the hands associated with the use of botulinum toxin-A injections for hyperhidrosis: a case report and review of the literature. J Plast Reconstr Aesthet Surg 2009;62:e274-6.

31. Choi WH, Song CW, Kim YB, Ha CS, Yang GH, Woo HD, et al. Skel- etal muscle atrophy induced by intramuscular repeated dose of botulinum toxin type A in rats. Drug Chem Toxicol 2007;30:217-27.

32. Tok F, Ozçakar L, Safaz I, Alaca R. Effects of botulinum toxin-A on the muscle architecture of stroke patients: the first ultrasonographic study. J Rehabil Med 2011;43:1016-9.

33. Kawano A, Yanagizono T, Kadouchi I, Umezaki T, Chosa E. Ultrasonographic evaluation of changes in the muscle architecture of the gastrocnemius with botulinum toxin treatment for lower extremity spasticity in children with cerebral palsy. J Orthop Sci 2018;23: 389-93.

34. Seynnes OR, Maganaris CN, de Boer MD, di Prampero PE, Narici MV. Early structural adaptations to unloading in the human calf muscles. Acta Physiol (Oxf) 2008;193:265-74.

35. de Boer MD, Maganaris CN, Seynnes OR, Rennie MJ, Narici MV. Time course of muscular, neural and tendinous adaptations to 23 day unilateral lower-limb suspension in young men. J Physiol 2007; 583(Pt 3):1079-91.

36. Chow RS, Medri MK, Martin DC, Leekam RN, Agur AM, McKee $\mathrm{NH}$. Sonographic studies of human soleus and gastrocnemius muscle architecture: gender variability. Eur J Appl Physiol 2000; 82:236-44. 